m/S

médecine/sciences $1995 ; 11: 1219-23$

\title{
LA FACULTÉ DE MÉDECINE DE L'UNIVERSITÉ CATHOLIQUE DE LOUVAIN
}

\section{Nicole Boisacq- Schepens Léon Cassiers}

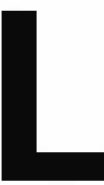

a Faculté de Médecine regroupe plusieurs Écoles dont celles de Médecine privilégiée dans cette présentation -, de Médecine Dentaire, de Pharmacie, et de Santé Publique situées à Woluwe (faubourg de Bruxelles)* et par ailleurs l'Institut d'Education Physique et de Réadaptation installé à Louvain-laNeuve. A ces structures d'enseignement et de recherche sont associées les Cliniques Universitaires Saint-Luc et Mont-Godinne auxquelles se rattache progressivement un important réseau d'institutions hospitalières. Après un survol de l'histoire de la Faculté de Médecine, cet éditorial tente d'en décrire la triple mission : enseignement, recherche, service. L'image instantanée ainsi produite se veut une vue d'ensemble - d'allure impressionniste - dont tous les détails ne peuvent être donnés ici. A titre d'exemple, les contributions scientifiques rassemblées dans ce numéro, dont le Professeur G. Rousseau a bien voulu assurer la coordination, résument quelques-uns des centres d'intérêt de notre École de Médecine dans le vaste champ de la recherche expérimentale.

* D'où l'appellation de notre campus : Louvain-en Wolutwe.

\section{Une Faculté marquée par I'Histoire}

Dès sa fondation, en 1425 à Leuven, l'Université Catholique de Louvain (UCL) a créé une Faculté de Médecine, bien modeste avec ses deux professeurs! Depuis ces débuts lointains, ses professeurs se sont consacrés non seulement à l'enseignement, mais aussi à la recherche. L'histoire de notre Faculté offre ainsi une galerie de personnages qui jalonnent l'histoire même de la médecine tels Érasme, Vésale, Van Helmont et, plus proches de nous, Schwann et Van Gehuchten pour ne citer que les plus célèbres, sans oublier C. de Duve à qui la découverte des lysosomes valut le prix Nobel de Physiologie et de Médecine en 1974. Dès les débuts, l'enseignement comportait des " choses naturelles" (anatomie, physiologie, hygiène) et des " choses préternaturelles » (pathologie, thérapeutique): cette distinction ne préfigure-t-elle pas le programme actuel centré d'abord sur les sciences précliniques et ensuite sur la formation clinique?

Par décret du 4 brumaire de l'an 6 (25 octobre 1797), l'UCL fut fermée mais, grâce à une poignée de professeurs, ses portes furent réouvertes dès 1817 comme université d'État. En fait, c'est en 1834 que l'UCL libre fut officiellement réinstallée avec une nouvelle Faculté de Médecine com- 
portant neuf professeurs et trentecinq élèves! On est bien loin du développement actuel de notre Faculté qui compte quelque 5000 étudiants représentant $24 \%$ de la population estudiantine de l'UCL et 374 membres du personnel académique et scientifique ; en outre, on y compte 1750 étudiants étrangers (dont 500 Européens environ) provenant de 70 pays.

L'explosion récente des progrès scientifiques laisse souvent l'impression que la vraie médecine, celle que nous connaissons actuellement, a commencé de novo il y a environ cinquante ans. Tout a changé, en effet, en un demi siècle à peine. Les professeurs mandarins sont devenus des chefs d'entreprise, ayant à gérer des services cliniques ou des équipes de recherche importantes. Les laboratoires dispersés d'antan, lieux de bricolages parfois géniaux, se sont mués en vastes plateaux techniques couverts d'appareillages sophistiqués. Les auditoires poussiéreux qui abritaient de petites cohortes d'étudiants sont devenus des amphithéâtres surpeuplés. L'hôpital vieillot où régnaient les bonnes sœurs s'est transformé en un lieu futuriste de haute technologie.

A ces changements qu'ont connus toutes les Facultés de Médecine, s'est ajoutée, pour la nôtre, l'épreuve d'un déménagement complet, entre 1970 et 1985 . Pour des motifs politiques linguistiques, en effet, la partie francophone de l'UCL a dû quitter la vieille ville de Louvain (Leuven) pour être transplantée à Louvain-la Neuve tandis qu'il a fallu reconstruire, partant de zéro, une nouvelle Faculté à Bruxelles : y réinstaller tous nos laboratoires, y créer un hôpital de 900 lits, y transplanter tout un campus avec étudiants et professeurs. Une expérience aussi dramatique pouvait être occasion de déclin ou source de renouveau. Nous pensons, avec l'ensemble des professeurs qui ont réalisé ce transfert et qui nous quittent actuellement peu à peu pour l'éméritat, qu'on a pu reconstruire sur le nouveau site, avec l'aide de l'université mère, une faculté qui réponde à toutes les exigences de la médecine moderne de pointe. En ou d'hôpital universitaire, cette implantation progressive témoigne de la présence de l'UCL à Bruxelles. Pour autant, ce développement ne s'est pas fait sans référence au passé. La tradition de notre Faculté est, et veut rester, proprement médicale. Même si la spécialisation technologique diversifie nos chercheurs, du cyclotron à la biologie moléculaire, cette tradition médicale veut que notre recherche reste centrée sur le malade et la santé. La recherche fondamentale reste articulée à la recherche clinique pour éviter que ne se crée une coupure entre le monde médical et le monde scientifique. D'où aussi la présence, dans notre Faculté, d'une École de Santé Publique forte, d'un intérêt pour les sciences humaines dans leurs dimensions médicales, et d'un Centre de Bioéthique. Plutôt que de concentrer tout l'effort de la Faculté sur le seul domaine biomédical de pointe, nous voulons couvrir les principaux domaines dans lesquels se joue aujourd'hui et se jouera demain la santé de la population.

Par une même fidélité à cette tradition médicale, nous essayons aussi de rester un lieu d'excellence de l'enseignement médical : nous formons près de la moitié des médecins francophones belges, et nous ne voulons pas nous soustraire à cette responsabilité envers la population.

Et l'avenir ? On peut estimer qu'il est lourd de défis aussi difficiles que ceux que la Faculté a dû rencontrer dans son passé. Le financement de la recherche par les pouvoirs publics, en Belgique francophone, est assuré notamment par le ministère fédéral de la Politique scientifique et par le ministère de l'Enseignement de la Communauté Française (via le Fonds National de la Recherche Scientifique). L'atout principal de cette organisation est de concentrer les fonds publics de la recherche dans les universités. Celles-ci trouvent, en outre, des ressources en hommes dans le financement de l'enseignement. A cela s'ajoutent diverses ressources provenant de fonds et mécénats privés, et des ressources croissantes auprès de l'Union Européenne. Cette multiplicité de provenances ne doit cependant pas faire illusion. L'investissement du pays dans la recherche ne fait, en effet, que diminuer par rapport au produit national brut. La Belgique consacre à sa recherche médicale, par habitant, dix fois moins d'argent que les ÉtatsUnis, et moins que chacun de ses voisins. Rien ne permet de prévoir le redressement de cette tendance, en Communauté Française tout au moins. A ne considérer que celle-ci, notre avenir peut paraître lourdement handicapé, malgré l'excellente rentabilité de l'argent consacré à la recherche que nos universités et notre pays ont pu réaliser jusqu'ici. Nous devrons donc nous tourner vers une coordination toujours meilleure de la recherche entre les universités francophones belges, en dépassant nos clivages politiques et particularistes. Dans le même mouvement, nous devons nous situer dans le réseau européen, développer nos accords actuels avec les laboratoires des autres pays, et les multiplier. $\mathrm{Si}$ nous restons capables de former de très nombreux doctorants, notre faiblesse porte sur notre capacité d'assurer leur avenir. Comme nous venons de le voir, d'importants défis - deux guerres, un transfert - ont pu être relevés, de manière satisfaisante. Dans la ligne de cette tradition, nous faisons confiance à nos jeunes générations actuelles et à venir pour relever le défi présent.

\section{Une Faculté tournée vers l'avenir}

\section{L'enseignement médical}

La Faculté est très attentive à la qualité de la formation scientifique et clinique qu'elle assure à ses futurs diplômés : un audit récent révèle les points forts et les points faibles de l'enseignement de l'École de Médecine qui s'est assignée de nouveaux objectifs brièvement rappelés ici.

\section{Les défis de la pédagogie médicale}

L'enseignement de la médecine pose des questions générales, communes à toute pédagogie universitaire, mais aussi des problèmes qui lui sont spécifiques. 
Le plus connu de ceux-ci tient à la masse des matières à enseigner. La médecine évolue avec une grande rapidité qui se traduit par des nouveautés incessantes, tant en sciences fondamentales qu'en clinique. Pour garantir la compétence du médecin, l'enseignant est toujours tenté d'enseigner trop. La pente est bien connue de surcharger les cours et de noyer les étudiants sous les matières à assimiler.

Le deuxième défi pédagogique tient au morcellement de la médecine. L'approfondissement des connaissances conduit fatalement à une spécialisation toujours plus poussée. Or le malade n'est qu'une seule personne, et toute pathologie locale retentit sur l'ensemble de l'organisme. En outre, la guérison dépend souvent des compensations que d'autres parties du corps, plus ou moins saines, pourraient apporter au déficit d'un organe. Il reste difficile, comme on le sait, pour un spécialiste de bien maîtriser toutes ces interactions qui font du sujet vivant une seule personne globale et il lui est, sans doute, encore plus difficile de l'enseigner.

Troisième défi que rencontre l'enseignement médical : la nécessité de combiner, chez le praticien, une formation aiguë à l'esprit scientifique avec une formation tout aussi poussée aux problèmes psychologiques et existentiels des malades. Quoi qu'on en pense, il n'est nullement évident d'enseigner ou d'acquérir en parallèle ces deux types de formation, et moins évident encore d'apprendre à les mettre en œuvre simultanément sur le terrain.

A ces trois défis majeurs de toute pédagogie médicale s'en ajoutent quelques autres, à peine moins importants, et tout aussi difficiles à relever : on attend du médecin qu'il soit compétent en éthique, en santé publique, en éducation à la santé, en économie, etc.

\section{L'évaluation de l'École de Médecine}

Ces défis pédagogiques ne sont certes pas nouveaux, mais la difficulté à les relever va croissant. Les enseignants de la Faculté sont habitués à soumettre leurs recherches à la critique interne et externe. Ils se sont tout naturellement demandé pourquoi ne pas procéder de même à propos de leur enseignement, même si cette démarche est plus originale en Europe, parce que loin de nos traditions académiques.

Or, une tradition existe en la matière outre-Atlantique. Les facultés de médecine nord-américaines doivent, pour rester agréées par leur association, procéder à une évaluation septennale de leurs enseignements. Notre Faculté a donc tout naturellement choisi leur méthodologie largement éprouvée.

Celle-ci comporte une démarche en deux temps. Le premier, qui est aussi le plus long et le plus approfondi, est un temps d'auto-évaluation. Les enseignants et les étudiants y sont conviés à réfléchir de manière critique sur leurs activités pédagogiques, notamment à l'aide de questionnaires détaillés (réadaptés aux réalités européennes). Le rapport qu'ils en tirent est adressé aux évaluateurs externes. Nous l'avons complété par une enquête auprès de nos anciens médecins, des promotions 1985 et 1989.

Le deuxième temps de la démarche met en scène les auditeurs externes. Trois mois après réception du rapport interne, six doyens ou anciens doyens de Faculté de Médecine (Canada, États-Unis, France, Tunisie et Université Belge néerlandophone) ont passé une semaine dans la Faculté, interrogeant un très grand nombre de personnes pour se forger une opinion aussi exacte que possible sur le fonctionnement de l'enseignement. Début mai 1994, ils ont déposé leur rapport. Ce dernier a fait l'objet d'une première analyse par une trentaine d'enseignants de la Faculté au cours d'une fin de semaine de travail, puis il a été distribué aux membres de la Faculté.

\section{Les réforines}

Si ce rapport reconnaît la qualité des médecins que nous formons, il propose une série de réformes dont le sens général est d'augmenter la participation active de l'étudiant à sa propre formation. En outre, il souligne l'insuffisance (générale en Europe) de la formation à rencon- trer les dimensions psychiques et sociales des maladies. Il apporte de nombreuses suggestions intéressantes en vue de moderniser les techniques pédagogiques utilisées.

Depuis septembre 1994, suivant en cela la dynamique de changement instaurée par la commission permanente de l'enseignement de l'École de Médecine, les enseignants se sont attelés à concrétiser ces recommandations dans un aggiomamento en profondeur. Celui-ci demande d'autant plus d'imagination que notre pays ne connaît pas encore le numerus clausus des étudiants en médecine, qui restent particulièrement nombreux. Néanmoins, l'esprit a changé. De trop passive qu'elle était, comme encore souvent en Europe, l'étude de la médecine devient l'entreprise propre de chaque étudiant. Le manque de cadres lui-même, en attendant un numerus clausus toujours espéré, se compensera en mobilisant les étudiants aînés et les futurs spécialistes dans le monitorat des plus jeunes. Le recours à l'autoapprentissage informatique, déjà présent pour l'un ou l'autre cours, va être étendu. La Faculté de Médecine espère ainsi rejoindre, quant à la qualité de sa pédagogie, le niveau d'excellence de ses chercheurs.

\section{La recherche}

Les thèmes de recherche abordés à la Faculté de Médecine couvrent un large spectre qui va des problèmes biomédicaux les plus fondamentaux aux sciences humaines et à la santé publique. Cette variété traduit la richesse de la Faculté en personnalités capables de rassembler les chercheurs en équipes de taille modeste ou importante. Certaines de ces équipes perpétuent l'héritage scientifique de leur fondateur (X. Aubert, C. de Duve, M. De Visscher, J.F. Heremans, H.G. Hers, J.P. Hoet, M. Lechat). D'autres sont plus récentes et ont pu être constituées avec le soutien de l'Université, qui veut assurer le renouveau tout en maintenant les traditions de qualité. Même si certains programmes de recherche ont un caractère très fondamental tandis que d'autres relèvent de la recherche clinique appli- 
quée, les deux approches sont de plus en plus souvent combinées parce qu'elles se fécondent mutuellement. Dans tous les départements de sciences fondamentales, les laboratoires accueillent fréquemment des médecins cliniciens qui viennent s'y former à la recherche avant de retourner dans les services hospitaliers, où ils lancent leurs propres projets en collaboration avec leur ancien laboratoire d'accueil. La place manque pour décrire le panorama complet de ces recherches. Comme les articles de ce numéro, notre énumération se limitera donc à l'École de Médecine.

- Les recherches dans le domaine de l'endocrinologie et du métabolisme ont toujours occupé une place importante dans plusieurs départements de la Faculté. Mentionnons les travaux du groupe de E. Van Schaftingen sur le contrôle du métabolisme des hydrates de carbone et plus particulièrement sur la régulation de la glycolyse par le fructose-2,6-biphosphate et par la glucokinase, de même que les travaux de G. van den Berghe sur l'influence de dérivés puriques sur le métabolisme hépatique. C'est en étroite collaboration que se poursuivent les recherches du groupe de G. Rousseau sur les mécanismes par lesquels les facteurs hormonaux et tissulaires contrôlent l'activité des promoteurs de gènes dans les cellules eucaryotes et de celui de L. Hue sur les mécanismes de synthèse et de dégradation du fructose-2,6-biphosphate ainsi que sur la lipogenèse et la synthèse de glycogène (voir l'article de L. Hue et al.). Dans une même perspective s'inscrivent les recherches de l'équipe de J.C. Henquin sur les mécanismes cellulaires du contrôle de la sécrétion d'insuline (voir l'article de J.C. Henquin et P. Gilon) ainsi que sur les mécanismes de l'insulinorésistance et ses possibilités de traitement. Sont également liés au diabète les travaux de J. Rahier sur la pathologie du pancréas endocrine et de R. De Hertogh sur les fotopathies. Dans un domaine connexe, le groupe de J.M. Ketelslegers étudie les répercussions de troubles nutritionnels sur le fonctionnement de l'hypophyse et le contrôle
J.P. Thissen et al.). Divers aspects de l'endocrinologie de la reproduction (K. Thomas) et de la thyroïdologie (J.F. Denef, C. Beckers) sont aussi abordés dans des études fondamentales et cliniques.

- De nombreuses facettes de la cancérologie retiennent l'attention des chercheurs de la Faculté. Le groupe de T. Boon a identifié et caractérisé des antigènes de surface exprimés par certaines cellules cancéreuses chez la souris et chez l'homme. Il étudie les possibilités thérapeutiques que pourrait offrir une immunisation de patients cancéreux contre ces antigènes (voir l'article de T. Boon et $a l$.). Plusieurs équipes (A. Ferrant, Ph. Martiat, M. Symann), travaillant à l'interface de la clinique et des recherches expérimentales, consacrent leurs efforts à l'amélioration de l'efficacité et à la diminution des inconvénients du traitement des hémopathies malignes. Enfin, le traitement de diverses tumeurs par neutronthérapie et protonthérapie a été développé par A. Wambersie et son groupe.

- Dans le laboratoire d'immunologie de P. Masson, sont étudiées les caractéristiques particulières de la réponse à certains virus (J.P. Coutelier) et les mécanismes de défense des muqueuses (J.P. Vaerman). Le groupe de J. van Snick est connu pour la découverte de certaines interleukines et de leur mode d'action (voir l'article de J.C. Renauld). Nos cliniques universitaires sont un centre de référence pour la transplantation d'organes. Rien d'étonnant dès lors que l'étude des mécanismes de rejet des greffes et l'utilisation de xénogreffes fassent l'objet d'études conjointes par des immunologistes et des chirurgiens (H. Bazin, P. Gianello).

- L'étude de la biologie cellulaire transcende les disciplines. La morphologie quantitative est à la base des travaux de P. Baudhuin et P. Courtoy sur l'endocytose (voir l'article de P. Cupers et al.). Le groupe de Th. Godfraind est connu pour ses travaux sur la pharmacologie des canaux calciques. Il étudie aussi, de même que le groupe de J.M. Gillis, le rôle du calcium dans le couplage stimulation-contraction. La régulation des récepteurs et de leurs systèmes de transduction dans les cellules nerveuses est étudiée par J.M. Maloteaux.

- Les recherches de G. Cornelis et de F. Opperdoes concernent deux aspects de la pathologie infectieuse. Le premier (voir son article), en utilisant le modèle de Yersinia enterocolitica, tente de démonter les mécanismes mis en œuvre par les bactéries pour infecter leur hôte. Le second (voir son article), à partir d'études sur la glycolyse chez les parasites, tente de modéliser des médicaments contre les trypanosomes et les leshmanies.

- Plusieurs équipes s’intéressent plus particulièrement à l'appareil locomoteur : celle de Ch. Delloye met au point des techniques de greffe osseuse et ostéochondrale, celle de G. Maréchal caractérise les propriétés des diverses isomyosines et celle de Ch. Nagant étudie les affections métaboliques du squelette, les maladies du cartilage et la pathogenèse des rhumatismes systémiques. Signalons également les travaux de G. Vaes et Y. Eeckhout sur la matrice extracellulaire, en particulier les métalloprotéases (voir l'article de E. Marbaix et al.).

- Dans le domaine des neurosciences se situent les travaux du groupe d'A. Roucoux sur les processus sensori-moteurs impliqués dans le guidage du regard et du mouvement de la main (voir l'article de A. Roucoux et M. Crommelinck) : ces études débouchent notamment sur la modélisation des mouvements du regard visant à développer des systèmes artificiels d'orientation des capteurs visuels. Par ailleurs, la technique du PET scan est progressivement appliquée dans le contexte des mêmes projets, tout comme dans l'approche de la réorganisation fonctionnelle du cortex cérébral en cas de cécité étudiée par C. Veraart et collaborateurs. Plus proche de la clinique, l'équipe de J.N. Octave étudie l'expression de protéines impliquées dans la maladie d'Alzheimer (voir l'article de J.N. Octave et al.) et celle de D. Rodenstein étudie les mécanismes de l'apnée du sommeil tandis que l'unité de Ph. Évrard conduit d'intéressantes recherches sur les aspects neurobiologiques, neuropa- 
thologiques et neuropsychologiques du développement cérébral. En psychiatrie clinique, les équipes dirigées par L. Cassiers ont fortement développé les dimensions sociales et psychothérapeutiques des traitements. Elles ont introduit en Belgique la thérapie familiale, développé de nouveaux modèles de prise en charge des toxicomanies (J.P. Rousseaux) et des urgences (M. Declercq), et conduisent des études sur les facteurs prédictifs de psychopathologie chez les grands prématurés (J.Y. Hayez). Parallèlement a été mené un important travail de réflexion épistémologique (Ph. Meire).

- A la frontière des recherches fondamentales et cliniques se situent les travaux en pathologie cardiovasculaire. Ils concernent surtout les cardiopathies ischémiques et l'insuffisance cardiaque (J.M. Detry). Le concept de l'hibernation myocardiaque a été particulièrement bien étudié grâce à la tomographie par émission de positrons dans l'équipe de J. Melin (voir l'article de J.L. Vanoverschelde et al.) . Dans le même domaine s'inscrivent les travaux de J. Donckier sur les rôles physiopathologiques de l'hormone natriurétique cardiaque et de l'endothéline. Mentionnons également les travaux de J. Donnez sur l'endométriose et le traitement chirurgical de la stérilité tubaire, ceux de Ch. Van Ypersele sur diverses maladies rénales et leur traitement, ceux de J.P. Buts sur le développement postnatal du tube digestif et ceux de A. Geubel sur la pathologie hépatique.

Enfin, dans le cadre de l'École de Santé publique, les travaux de l'équipe de $R$. Lauwerys font référence dans plusieurs secteurs de la toxicologie industrielle, en rapport, notamment, avec divers métaux ; en outre, la problématique du système de santé est analysée par le groupe de D. Deliège (voir leur article) tandis que d'importants subsides (Organisation Mondiale de la Santé, Union Européenne) sont octroyés à différentes études dans le domaine de l'épidémiologie et de la planification sanitaire.

\section{Les services}

En quelques mots, l'ouverture de la Faculté à la société se concrétise par son engagement vis-à-vis des services hospitaliers universitaires, et extra-universitaires, notamment les CREMEC (centres régionaux d'enseignement par les stages, y compris auprès des médecins généralistes) et plus récemment encore dans la CRHU (commission du réseau hospitalier universitaire) qui constitue un important lieu de collaboration et d'entraide au niveau de la Communauté médicale francophone de Belgique. A travers ces collaborations, la Faculté entretient des contacts proches avec la médecine telle qu'elle se pratique sur le terrain, afin d'en faire profiter son enseignement. En outre, de nombreuses collaborations existent avec diverses sociétés scientifiques du pays aux fins d'assurer un enseignement continu postuniversitaire de qualité pour les médecins en fonction.

Au-delà de nos frontières, et plus particulièrement dans les pays en voie de développement, plusieurs réseaux de relations ont vu le jour à l'initiative, notamment, de MEDEVUC: on rappellera le soutien apporté par l'UCL à la Faculté de Médecine de Cotonou (Bénin) et, très récemment, son appui à une collaboration Sud/Sud entre cette dernière et Bukavu pour faire face aux incidents dramatiques dans cette région de l'Afrique centrale. Un autre réseau de relations existe également avec des pays d'Amérique latine, tantôt pour aider leurs universités, tantôt pour développer un soutien aux populations des bidonvilles, en particulier dans l'éducation des enfants.

Pour conclure, cet éditorial a esquissé en quelques traits l'horizon de la Faculté de Médecine de l'Université catholique de Louvain, enracinée dans son prestigieux passé et largement ouverte à d'importants développements actuels, en dépit de difficultés budgétaires, communes d'ailleurs à toutes les institutions universitaires. Ce faisant, il invite le lecteur à s'intéresser à quelques-unes des recherches originales menées à l'École de Médecine et présentées dans ce numéro

\section{TIRÉS À PART}

N. Boisacq-Schepens. 